\title{
Solar Activity and Geomagnetic Storm Effects on GPS Ionospheric TEC over
} Ethiopia

\author{
Aragaw Msganaw ${ }^{1}$, Gebiregiorgis Abraha ${ }^{2 *}$ and Tsegaye Kassa ${ }^{3}$ \\ ${ }^{1}$ Department of Physics, Assosa University, Assosa, Ethiopia. \\ ${ }^{2}$ Department of Physics, CNCS, Mekelle University, Mekelle, Ethiopia (*ggabraha@gmail.com). \\ ${ }^{3}$ Department of Physics, Bahir Dar University, Bahir Dar, Ethiopia.
}

\begin{abstract}
Ionospheric GPS total electron content (TEC) is an important parameter to monitor for possible Space Weather impacts. The effects of solar activity on TEC at low latitude stations with geographic locations (latitude, longitude) of Addis Ababa $\left(9.04^{0} \mathrm{~N}, 38.77^{0} \mathrm{E}\right)$ and Bahir Dar $\left(11.6^{0} \mathrm{~N}, 37.36^{0} \mathrm{E}\right)$ in Ethiopia, East Africa in the year of 2015 around peak of solar cycle 24 has been carried out. The data from the two stations was used to study the diurnal, monthly and seasonal variations of TEC and its dependence with solar activity and space weather effects. These observations were investigated and further discussed with an analysis of Disturbance Storm Time (Dst) and Ap indices, solar radio flux (F10.7 cm) and sunspot number during the period of 2015. During the period of low or high sunspot number, that provided GPS ionospheric TEC builds up slowly or quickly. The obtained results reveal TEC undergoes diurnal and seasonal variations, daily variation of TEC value at both stations sharply increases to its peak from 0900 -1500 UT and decreases around 1600 - 0700 UT. Seasonal variations showed that TEC maximizes during the equinoctial months and least in summer over the two stations. In all seasons the maximum value of TEC in Addis Ababa is higher. The effects of geomagnetic storms on TEC values have been found negative and positive output.
\end{abstract}

Keywords: Solar activity, GPS, Ionosphere, TEC, Geomagnetic storm, Ethiopia.

\section{INTRODUCTION}

As mankind has become increasingly dependent on technology, understanding of the effects of the Space Physics phenomena on human technology, termed Space Weather, has become increasingly necessary (Jakowsky et al., 2002). The Sun's violent activity and many unexpected and unpredictable events taking place on its surface suggest that we should prepare for the worst. The changes on the Sun cause effects in space, in the atmosphere, and on Earth's surface. Most aspects of Space Weather affect us to some extent. The more our society becomes dependent on technology and the more we utilize space, the more we are affected by Space Weather. Its effects are highly relevant to our daily life on Earth. Technology dependence has become increasingly vulnerable to adverse Space Weather. Although the technology was affected only by the most severe ones in the past, nowadays our society demands more sophisticated technology, therefore may become more vulnerable even at less severe disturbances. 
Methods for forecasting Space Weather have thus generated considerable research interest. Huge explosions of magnetic field and plasma from the Sun's corona, known as coronal mass ejections (CMEs), could one day produce extremely powerful geomagnetic storms striking Earth with enormous power, showing no mercy to our planet. CMEs are the most dangerous and destructive Space Weather events (Denton et al., 2006). Geomagnetic storm is a worldwide disturbance of the Earth's magnetic field, associated with solar activity. The studies of these worldwide disturbances of Earth's magnetic field are important in understanding the dynamics of solar-terrestrial environment.

The increase in the density of the atmosphere as we go further down to the surface of the Earth and the decrease in the intensity of photons as it bombards the neutral atoms and the availability of different atoms and molecules at different heights from the Earth's surface forms a large scale vertical layer of ionization within the ionosphere. This makes the ionosphere to exist as vertically stratified partially ionized plasma state from at about 60-1000 km from the surface of the Earth (Kelly, 1989).

The electron density distribution over this region is, therefore, very crucial for satellite communication and navigation systems. However, the distribution of these electrons over the ionosphere exhibits complicated structure especially during increased solar activity. Hence, exploring the ionosphere is of utmost interest due to the complexities associated with the region (Tsurutani, 2000; YU et al., 2009; Michael, 2009). Nowadays, several ionospheric delay models can be used for real-time or post processed applications. Among these ionospheric delay models, the Klobuchar model, which is driven by broadcast ephemeris, is widely used by GPS users (Ke Su et al., 2019; Klobuchar, 1987).

Due to insufficient ionospheric devices the physics of the ionosphere in Ethiopia has not been studied well. Now a day few ground based GPS receivers have been installed and as a result it gives a good opportunity for us to observe effects of solar activities on GPS ionospheric TEC. The computation of reliable vertical total electron content (VTEC) of the ionosphere is at the same time an useful and challenging goal. Useful: because in both Science and Technology fields, they can provide valuable information concerning Space Weather Events, empirical model predictions, and user navigation improvement, among others (Adolph, 1985; Coster and Komjathy, 2008). 
TEC is obtained from the dual frequency code measurements using the relation (Bagiya et al., 2011; Hintsa and Gebregiorgis, 2017; Yizengaw et al., 2007).

$$
\begin{aligned}
& S T E C=\left[\frac{2}{K} \frac{f_{2}^{2} f_{1}^{2}}{f_{1}^{2}-f_{2}^{2}}\right]\left(P_{2}-P_{1}\right) \\
& S T E C=\left[\frac{2}{K} \frac{f_{2}^{2} f_{1}^{2}}{f_{1}^{2}-f_{2}^{2}}\right]\left(L_{1} \lambda_{1}-L_{2} \lambda_{2}\right)
\end{aligned}
$$

Where, $f_{1}$ and $f_{2}$ are GPS signal frequencies and are equal to $1.57542 \mathrm{GHz}$ and $1.2276 \mathrm{GHz}$ respectively, and $\mathrm{K}=80.62 \mathrm{~m}^{3} \mathrm{~s}^{-3}$ is a constant that relates plasma frequency to electron density. Where, $\lambda_{1}$ and $\lambda_{2}$ are wavelengths corresponding to $f_{1}$ and $f_{2}$. In addition to that if we assume there are no lateral or horizontal electron density gradients, the vertical TEC (VTEC) can be simply mapped to the Slant TEC(STEC) as follows.

$$
S T E C=\frac{V T E C}{\cos \aleph}
$$

Where, $\boldsymbol{N}$ is the satellite zenith angle at the point of intersection of the line of sight with that of spherical single layer atmosphere Piercing point (Abdullah et al., 2009).

The TEC measurements obtained from dual frequency GPS receivers are one of the most important methods of investigating the Earth's ionosphere. Because of the sensitivity of the science as stated above and the insufficient studies about ionospheric variability in Ethiopia, East Africa, we are motivated to investigate effects of solar activities and geomagnetic storms on GPS ionospheric TEC over Ethiopia by using Addis Ababa and Bahir Dar GPS stations in the year of 2015, which is around peak of solar cycle 24.

Due to insufficient ionospheric devices the physics of the ionosphere in the Ethiopian sector has not been studied well. Now-a- days few ground-based GPS receivers have been installed and as a result it gives a good opportunity for us to observe effects of solar activities on GPS ionospheric TEC. The computation of reliable vertical total electron content (VTEC) of the Ionosphere is at the same time a useful and challenging goal. It is useful because, in both Science and Technology fields, they can provide valuable information concerning space weather events, empirical model predictions and user navigation improvement, among others. Because of the sensitivity of the science and the insufficient studies about ionospheric variability in Ethiopia, East Africa, we are motivated to observe the effects of solar activities and space weather events by using ground-based GPS receivers over this sector with recent data. The main objective of 
this study is thus, to investigate effects of solar activities and geomagnetic storms on GPS ionospheric TEC over Ethiopia by using Addis Ababa and Bahir Dar GPS stations in the year of 2015, around peak of solar cycle 24 .

\section{DATA AND METHODOLOGIES}

One of the parameters that are used to study the ionosphere is the Total Electron Content (TEC). The variability of the ionosphere can be monitored using space and ground based probing techniques. Among the different techniques, the global positioning system (GPS), which uses a linear combination of observable two frequencies to remove the effect for positioning, is used in this study. The TEC measurements obtained from dual frequency GPS receivers are one of the most important methods of investigating the earth's ionosphere.

To study the solar activity, diurnal and seasonal variations and space weather events of ionospheric TEC over Ethiopia, we used Addis Ababa and Bahir Dar stations. GPS data from January to December 2015 for all days at Addis Ababa and Bahir Dar stations using a dual frequency $f_{1}(1575.42 \mathrm{MHz})$ and $f_{2}(1227.60 \mathrm{MHz})$ have been downloaded from UNAVCO data home page:(http:// facility.unavco.org/data/gnss/perm-sta.php). The data for Dst index is downloaded from world data center website: (wdc.kugi.kyot-u.ac.jp/dst_realtime/ presentmonth/index.html).

After all the data are received from the source, MATLAB programming with its code has been developed by importing the data. Using the MATLAB programming code the value of TEC in Addis Ababa and Bahir Dar stations for all days of each month and season including selected disturbed and undisturbed days and Dst index, solar radio flux and sunspot number data with the obtained Matlab program has been plotted.

\section{RESULTS AND DISCUSSION}

\subsection{Diurnal variations of TEC}

The diurnal variation of the electron density can be studied using TEC parameter. Figure 1 shows the diurnal variation of the TEC during 2015. The plots show TEC (TECU) versus universal time (UT) in hr at an elevation mask angle $30^{\circ}$ at Addis Ababa and Bahir Dar stations, in Ethiopia. TEC value has its maximum at noon time and is very low during night time. The TEC variation is not only time dependent it also depends on latitude. 


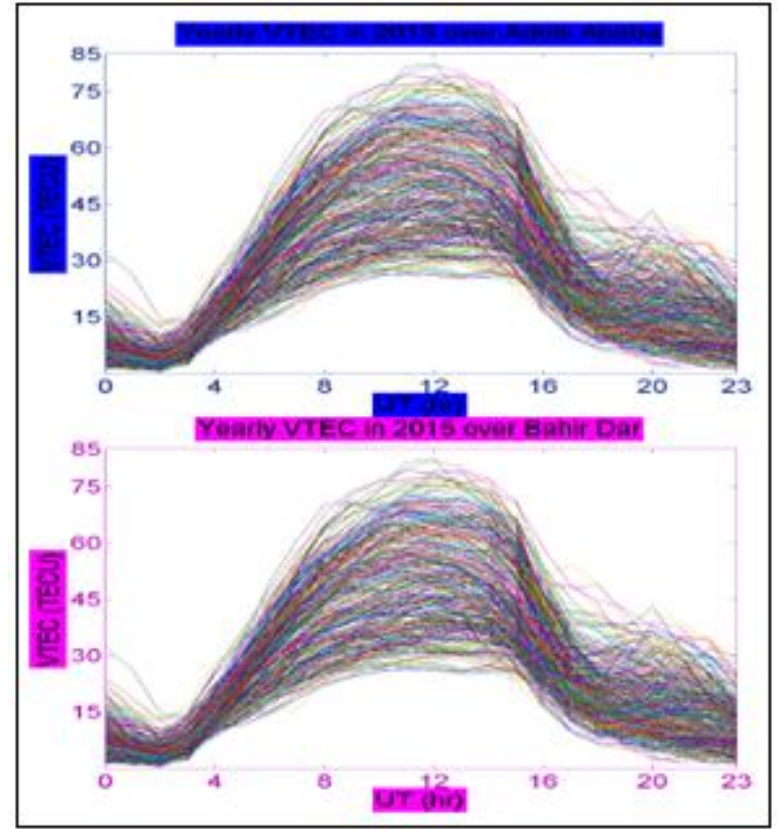

Figure 1. Mass plot of VTEC at Addis Ababa and Bahir Dar stations during 2015.

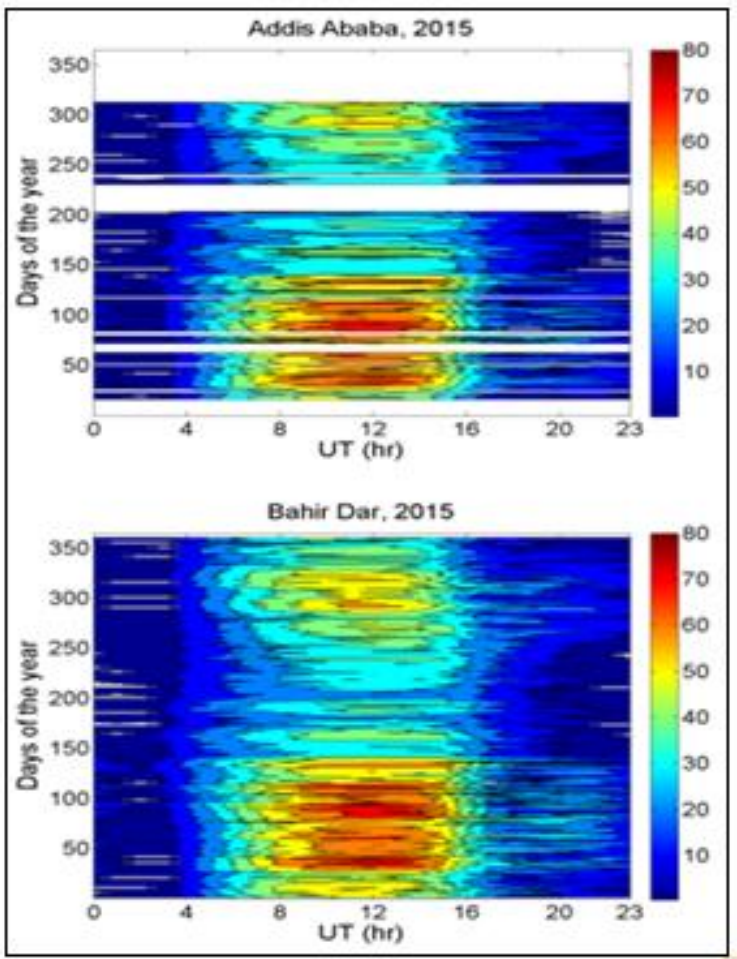

Figure 2. Contour plots of the diurnal variation in GPS-TEC measured at the study areas in Ethiopia. The white sections in the plots show unavailability of the data.

The variations with low at night time TEC value and peaks at noontime is the typical behavior of the ionosphere. As the sun is the main source of ionization most TEC variations are 
resulted due to the activity of the Sun. However, the modifications of the atmosphere from below cannot be neglected (Maruyama, 2003; Abdu and Brum, 2009). The diurnal patterns of TEC exhibits a steady increase starting about sunrise to an afternoon maximum and falls to attain a minimum just before sunrise. The diurnal characteristic of TEC has seasonal, solar activity, geomagnetic activity and latitudinal dependence.

As clearly indicated in figure 2, the magnitude of GPS TEC is generally high during day time at both locations. The day time GPS-TEC values are generally greater than the night time values. This can be attributed to the absence of solar radiation at night time. After this general observation we are interested to observe the overall and in depth phenomena happened in each months of days during the selected year for this study.

As observed from figure 2 due to the incompleteness of the data at Addis Ababa station during certain days of months, we presented the diurnal variations observed at Bahir Dar station dual frequency GPS-TEC data. The diurnal variation in TEC at Bahir Dar, Ethiopia exhibits many characteristics typical to low (equatorial) latitude ionosphere such as a TEC minimum at predawn and gradual increase with the time of day attaining a maximum in the afternoon and a gradual decrease after sunset. The daily peak occurs at about 0900 - 1500 UT (around 1200 UT) around low latitude (equatorial) regions. In equatorial regions or low latitude the daily variation peak TEC greatly depends on Sun's activity. The day to day variability of TEC is contributed by the various parameters like EUV, solar flares and CME which is consistent with previous results obtained earlier (e.g. Rama Rao et al., 2006).

Figures 3 to 8 show the mass plot of TEC diurnal variation for the different days of the year 2015. In all these plots the diurnal variations show a maximum occurring around 0900 1500 UT and short-lived minimum in TEC occurring around 0000 - 0800 UT and $1600-2300$ UT. Generally this temporal variation is depending on intensity of radiation coming from the Sun, since the intensity increases starting from sunrise and become maximum when Sun is overhead and comes to zero when sunset. As observed from figure 3, concentration of TEC is greater between $0900-1500$ UT, maximum TEC is observed during January 30 and during February $1-10,13,19-25$ and 28, 2015. Around January 4, 2015 it seems the least TEC measured. From figure 4, we observe that the concentration of TEC value observed in March is maximum between 0900 - 1500 UT. The peak TEC is measured on March 30 afternoon at 1100 
UT. In the morning and evening the amount of TEC is similar and less than the peak value. On the other hand maximum TEC around 80 TECU is measured in April.

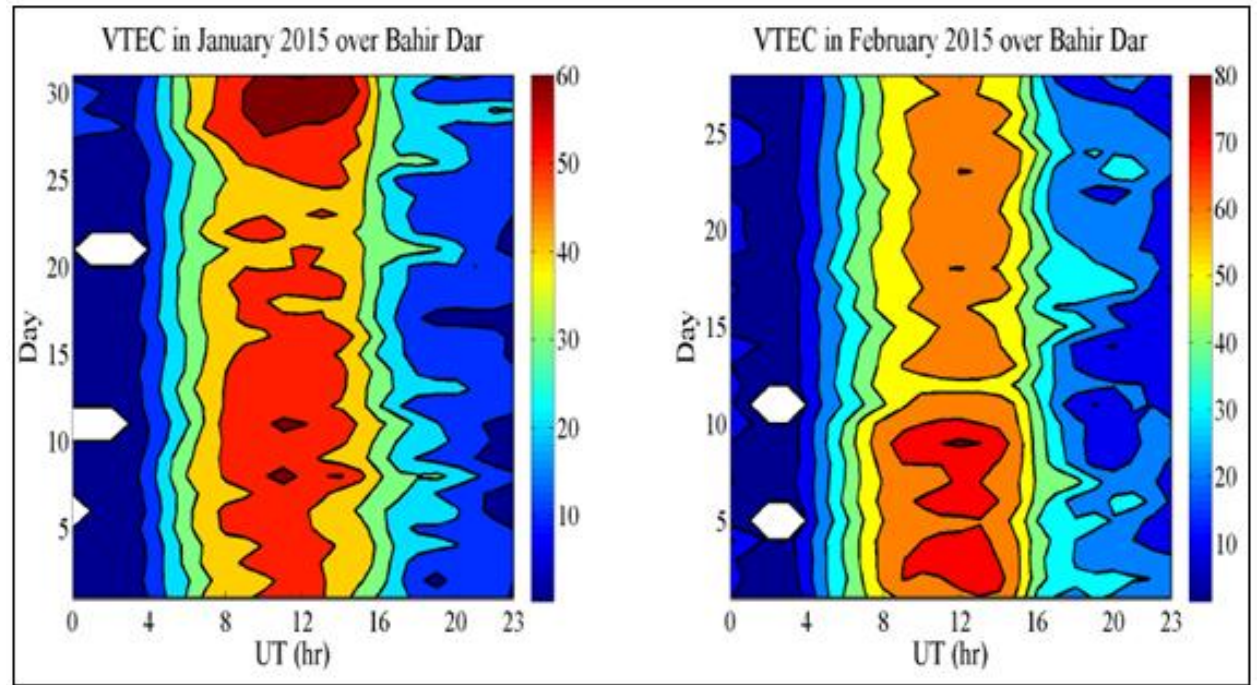

Figure 3. Diurnal variation of TEC observed at Bahir Dar station during January and February, 2015 .

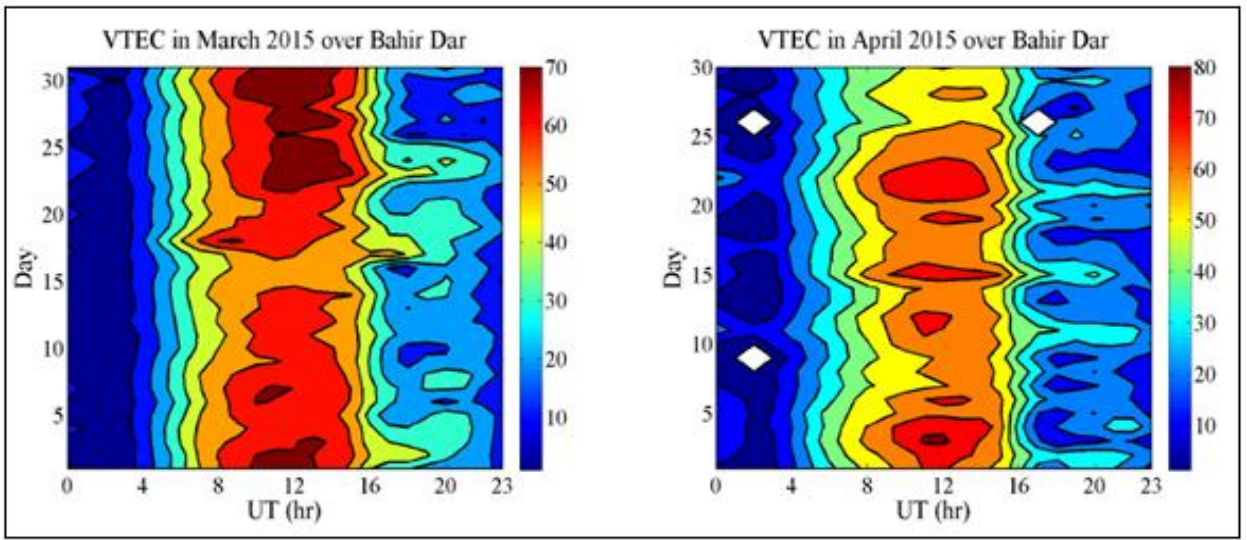

Figure 4. Diurnal variation of TEC observed at Bahir Dar station during March and April, 2015.

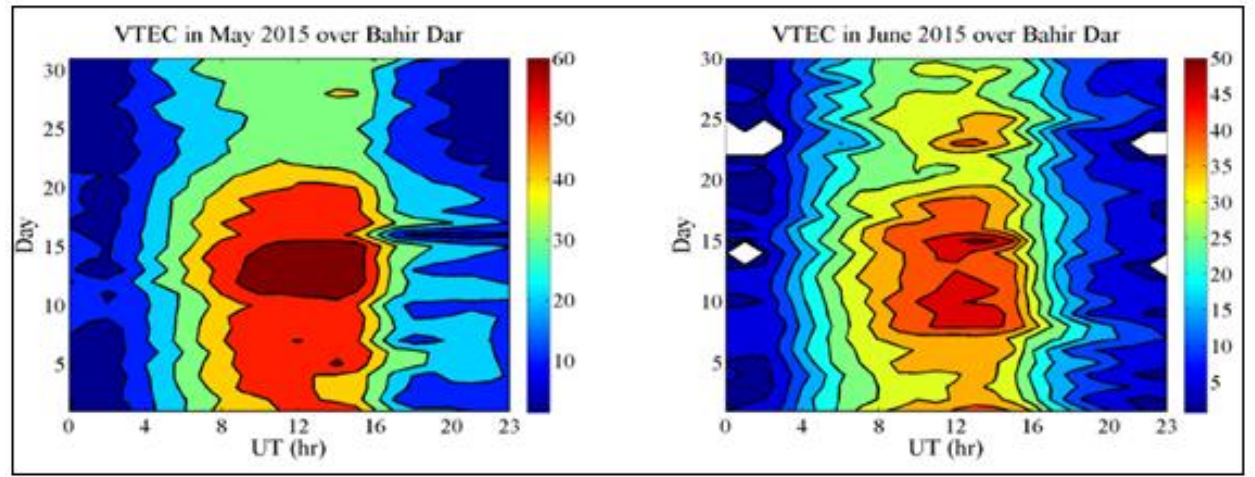

Figure 5. Diurnal variation of TEC observed at Bahir Dar station during May and June, 2015. 


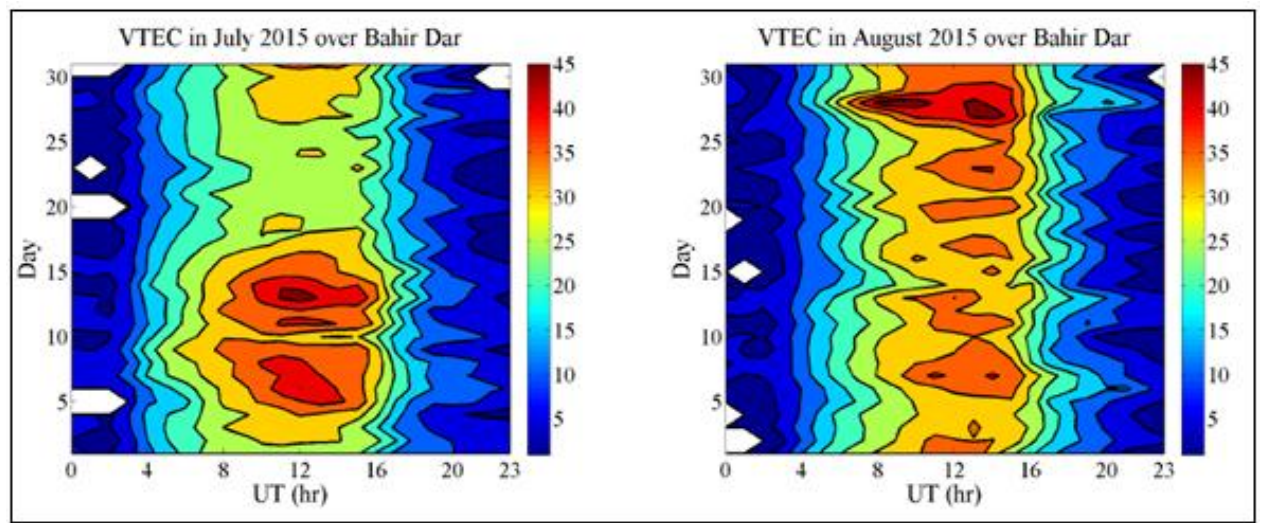

Figure 6. Diurnal variation of TEC observed at Bahir Dar station during July \& August, 2015.

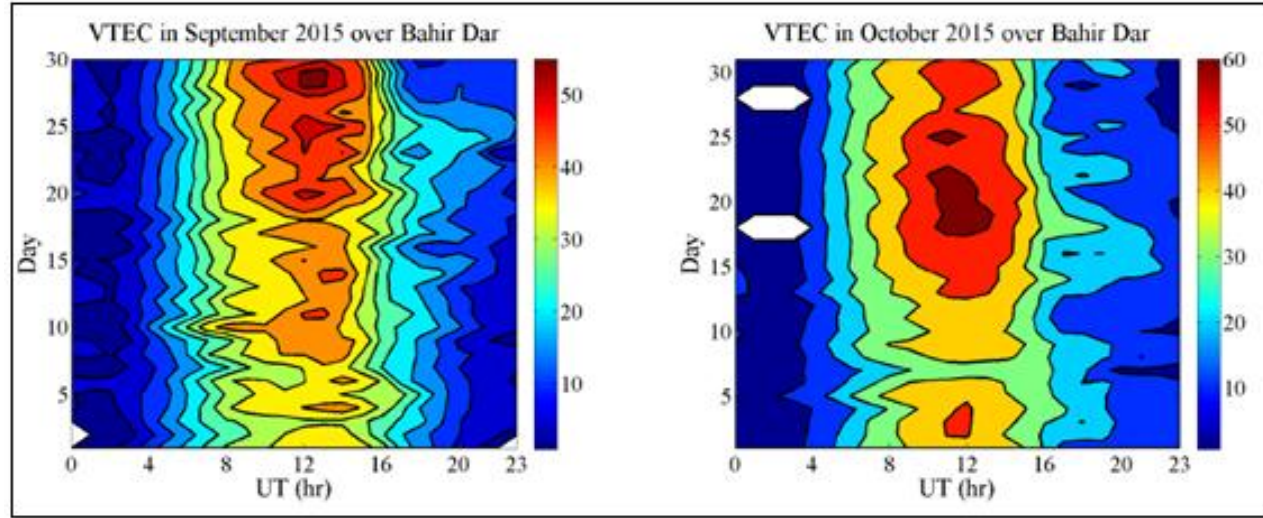

Figure 7. Diurnal variation of TEC observed at Bahir Dar station during September and October, 2015.

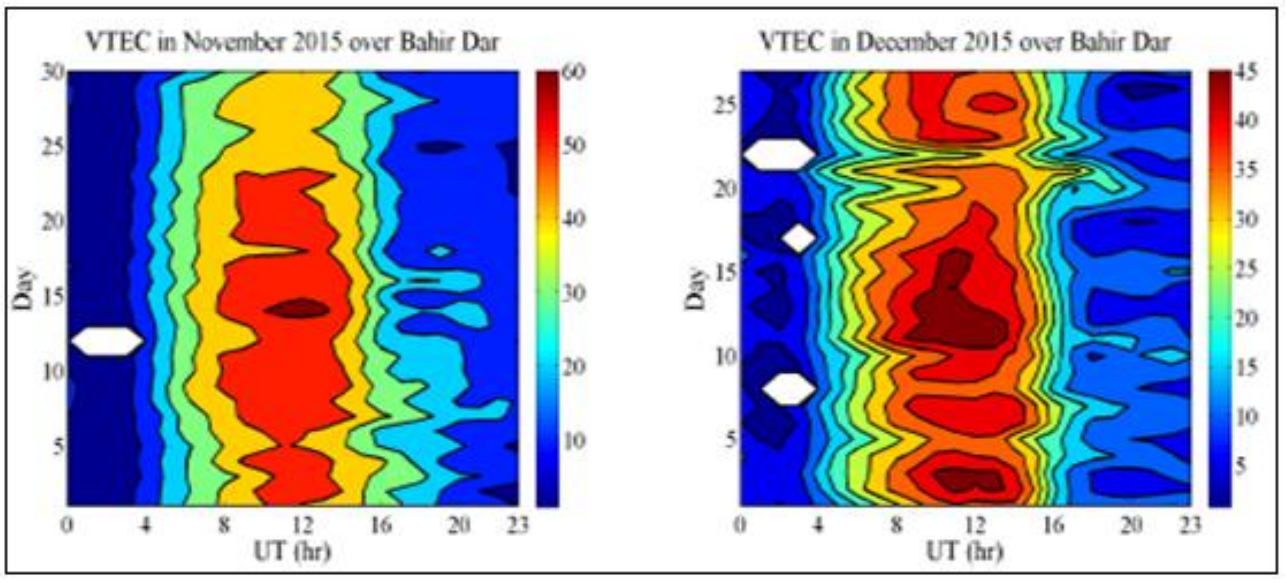

Figure 8. Diurnal variation of TEC observed at Bahir Dar station during November \& December, 2015.

In figure 5, Peak TEC of 68 TECU was observed in May 11 - 15, 2015. However, in June 5, 2015 exhibited TEC maximum peak value is about 39 TECU while on June 23, 42 TECU were recorded. On June 23, there is a geomagnetic storm occurred on Earth's magnetosphere and 
we observed its effect on TEC at the end of the next section. As shown from figure 6, the least peak TEC observed from the station during the days in the two months namely July and August. On July 5 - 8 and 11 - 14 and August 27 - 30, 2015 peak TEC were measured later after noon at 0800 - 1500 UT.

Based on figure 7 we can generalize that, the derived GPS ionospheric TEC value is maximum during late days of September and October as compared to June, July and August. In September 29 peak measured TEC were about 56 TECU, while on October 18, 64 TECU peak value was measured. From figure 8, we observe that during November similar peak TEC of magnitude 63 TECU was measured. From 2 - 3 and 11 - 16, December, 2015 at 0800 - 1500 UT the derived TEC value was maximum. From the indicated days somehow the solar activity was maximum then the GPS station drives strong TEC value. December month is an element of winter season, we expect the TEC value is intermediate so does the obtained result.

In all the figures 3-8, the TEC value of one day is different from the TEC value of another day of the same month. In all these contour plots the diurnal variations show a maximum occurring of TEC is found between 0900 - 1500 UT and short-lived minimum TEC occurring around 0000 - $0800 \mathrm{UT}$ and 1600 - $2300 \mathrm{UT}$. These day to day variations of TEC may be attributing to the change in activity of the Sun itself. This means that, sunspot number, geomagnetic activity and different particles coming from the Sun are different from day to day so that the TEC value varies accordingly. Moreover, the maximum VTEC is obtained at the day time near noon. As it is known, the intensity of the sun's radiation is influenced by the zenith angle of the Sun's rays striking the atmosphere. At noon time the intensity of light from the Sun is relatively large compared to the other hours of the day. Thus, photo-ionization processes are maximum and maximum TEC is obtained at noon time. During the night time the value of TEC is very small because electrons are lost as a result of the recombination processes.

\subsection{Monthly Variation of Ionospheric TEC}

Figure 9 shows that there are significant VTEC differences in both magnitude and pattern over 12 months period in the two stations in the buildup region, afternoon plateau and decay region. In all months the TEC value is maximum near noon and of all months the highest TEC is obtained around noon. In Addis Ababa station, the month of March recorded the highest peak TEC of 68.4 TECU at about 1100 UT followed by February and April with Peak TEC of 66.16 and 64.5 TECU at about 1200 and 1100 UT respectively. The least peak TEC of 37.4 TECU and 
36.67 TECU at about 1200 UT and 1300 UT was observed in July and August respectively. In Bahir Dar station, the month of March recorded the highest peak TEC of 69 TECU at about 1200 UT followed by April and February with maximum TEC value of 68 TECU and 67 TECU at about 1100 UT respectively, during the year 2015. The month of July recorded the least peak TEC of 32 TECU at about 1100 UT.

The variation of the monthly median TEC for the above mentioned period of 12 months for the two stations are as shown in the contour plots of figure 9. The features mentioned above are depicted very well in this figure. The minimum median TEC was registered from 1600 0700 UT except in the months of March, April and February where the periods of $1600-0700$ UT recorded moderate median TEC values during these months. The afternoon maximum was attained between the hours of 0900 and 1500 UT. The months of June, July and August recorded moderate median TEC values ranging from 36 to 38 TECU during the afternoon maximum. The highest median TEC values was recorded in the months of January, March, April, February and October with values ranging from 64 - 69 TECU. The exceptional peak value of the derived TEC for March, 2015 was because of it is an element of the equinoctial season. In more general case the peak TEC values reach first in Addis Ababa as compared to Bahir Dar.

Our ionospheric sector has been produced too much amount of free electrons during the day time. One can deduce from Fig. 9 there is strong TEC recorded throughout the whole months in the time about 0900 - 1500 UT. During February, March and April 2015 the magnitude of the TEC is better than the rest days of other months at 0000 - 2300 UT. The three months (March, April and February) TEC value during 2015 at both stations is extremely differ from other months TEC value. This is because March and April months are parts of equinoxes while February is winter season. As it is known and very well depicted in figure 10 the value of TEC is maximum during the equinoctial and winter seasons as compared to summer season. From TEC versus Universal time curve we deduce that TEC does not suddenly disappear, but gradually increasing and decreasing before 0600 and after 1600 UT respectively. From these temporal and diurnal variation of TEC, one can infer that unusual change comes from the Sun's activities such as sun spot number and solar flares which often accompany the fast coronal mass ejection and geomagnetic activity variation.

The day-to-day or month-to-month variability in TEC, which has also been observed by some authors (Yizengaw et al., 2007; Tyagi, 1974), may be due to the changes in the activity of 
the Sun itself and to the associated changes in the intensity of the incoming radiations and the zenith angle at which they are incident on the Earth's atmosphere. The reason for this phenomenon is that the hourly values of TEC can be altered by intensity of solar electromagnetic radiation and emission of particles from the Sun.

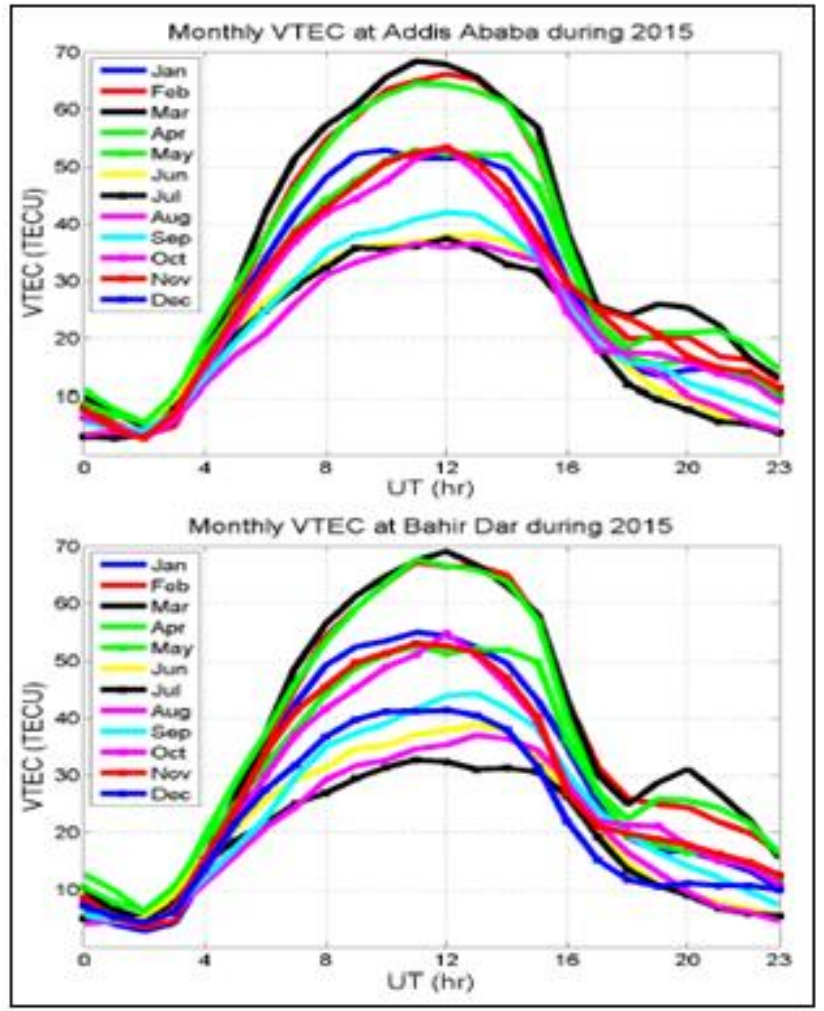

Figure 9. The median monthly Variation of TEC observed at Addis Ababa and Bahir Dar Stations during 2015.

\subsection{Seasonal Variation of Ionospheric TEC}

To study the seasonal variability of the ionospheric TEC, Lloyd's seasons as cited in (Rabiu et al., 2007; Rastogi et al., 2008 and references therein), in which the whole year is allotted in to three seasons; December or D-season (December, November, January and February), Equinox or E-season (March, April, September and October) and June Solstice or J-season (May, June, July and August) are used. During Equinox the Sun shines directly on the equator and the length of day and night is nearly equal and because of maximum photo-ionization, maximum TEC in the equatorial ionosphere is expected.

It is important to note that Earth does not move at a constant speed in its elliptical orbit. Therefore the seasons are not of equal length. The relative position of the Earth's axis to the Sun 
changes during the cycle of seasons. This phenomenon is the reason why the Sun's height above the horizon changes throughout the year. It is also responsible for the seasons through controlling the intensity and duration of sunlight received at various locations around the planet. So during solstice the Sun is not overhead at the equator, that means the light intensity that pass through the ionosphere above the equator is small compared to the equinox which leads to small value of TEC in the ionosphere which in turn produces different navigational and communication problems.

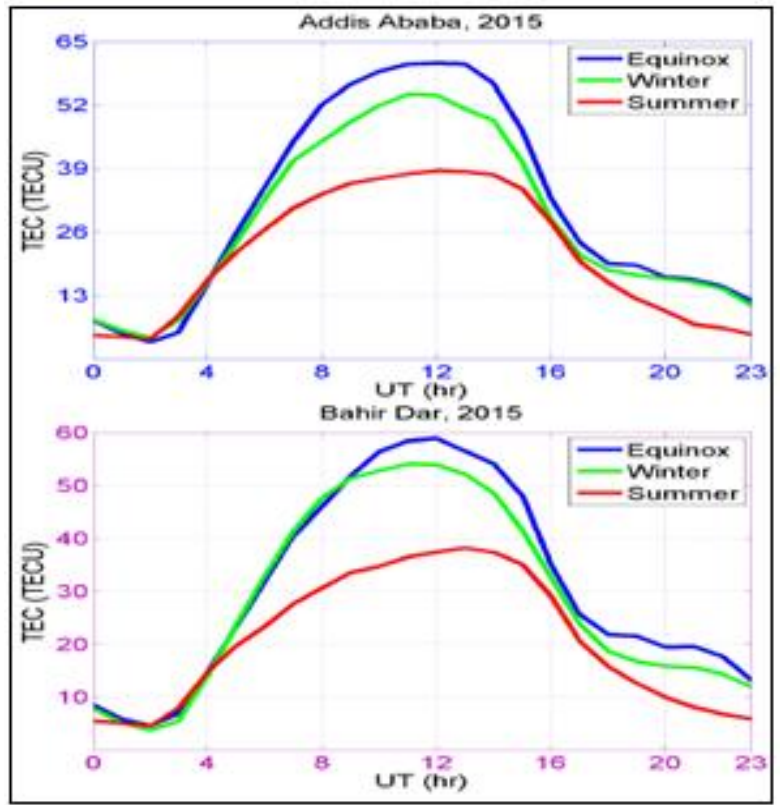

Figure 10. The median seasonal Variation of TEC observed at Addis Ababa and Bahir Dar Stations during 2015.

Accordingly, the occurrence time of the maximum /minimum ionospheric TEC values varies with seasons. The seasonal variation of ionospheric TEC for Addis Ababa and Bahir Dar stations since 2015 is stated in figure 10. The study of TEC variation at both stations has been grouped into three seasons as in the case stated before. Figure 10 shows the variation of TEC for the whole seasons for the two stations. As observed from this figure the TEC variations attained maximum during the afternoon with equinoctial months followed by winter for the two stations. Summer months recorded the least TEC amplitudes both in Addis Ababa and Bahir Dar.

The position of the Sun relative to the equator affects the ionization process in the ionosphere because the intensity of ionization depends on the solar zenith angle. That is when the sun is at different positions the solar zenith angle changes so the rate of production will be changed. 
When the Sun is at horizon the intensity reaching the equatorial ionosphere is relatively small compared to the intensity reaching at the equatorial ionosphere from the overhead Sun. The results of variation in median TEC over the low-latitude stations considered in this study showed that the variation of median TEC is dependent on seasons and local time of occurrence in both stations of Ethiopian low latitude regions. As shown in figure 11 in the whole seasons the maximum value of TEC leads first in Addis Ababa. The difference in VTEC over Addis Ababa and Bahir Dar is due to differences in geographical latitude.

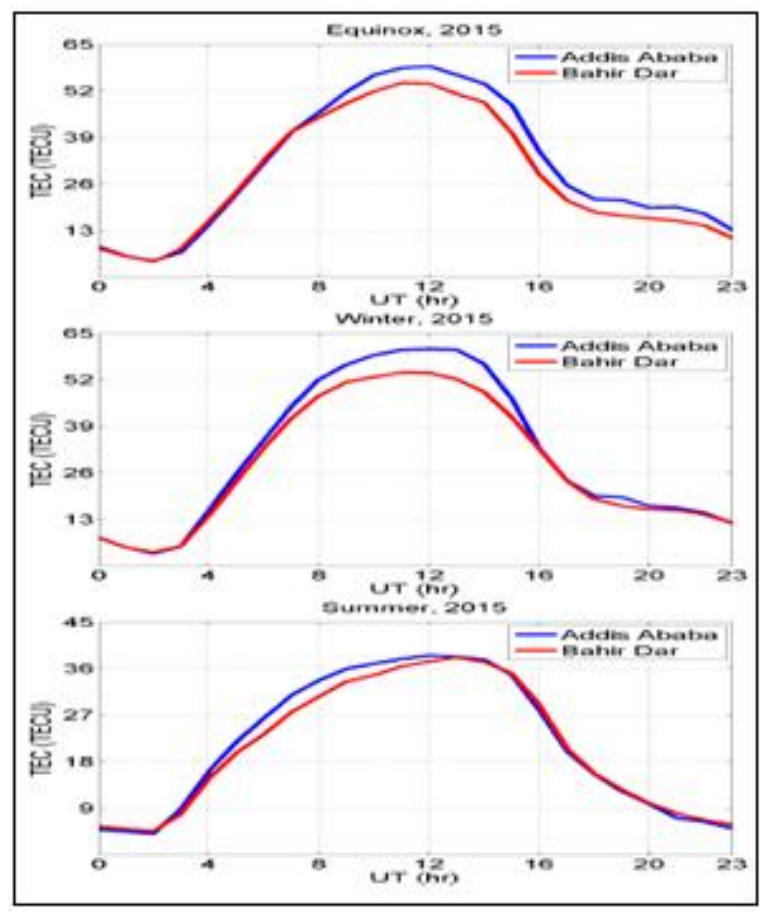

Figure 11. The dependence of seasonal variation of TEC on location.

\subsection{Solar Activity Dependence of TEC}

The Sun emits a wide spectrum of radiation along with high energy particles. When, radiation is absorbed, intensity is decreased. If intensity were not decreased or radiation were not absorbed one cannot exist over the Earth. This radiation increases during high solar activity and this has its own effect on Earth's ionospheric TEC, the leading indicator of Space Weather. To see the effect of this solar activity on the GPS-TEC, we demonstrate the solar activity indices (sunspot number and radio flux) data. The number of sunspots and solar flux changes with an 11-years period which is called the solar-activity cycle (solar cycle), as observed on figure 12. The demonstrated 
year 2015 is found around peak of solar cycle 24 as shown in figure 12 by red arrow. The peak of this solar cycle is indicated by the blue arrow on the stated figure which is 2014 .

Along with the sunspot number, the flux of the Sun's radio emission at a wavelength of $10.7 \mathrm{~cm}(2.8 \mathrm{GHz})$ is a useful indicator of solar activity relevant for ionospheric effect. Solar flux from the entire solar disk at a frequency of $2800 \mathrm{MHz}$ has been recorded by a radio telescope near Ottawa since February, 1947. The other quantity to take into account is the precipitation of particles most of them coming from the Sun (CMEs). The sunspot number index is also often called Wolf number in reference to the Swiss astronomer J. R. Wolf who introduced this index in 1848 (Arnold, 2007); sunspots and the solar-cycle. The Sunspot is annual, diurnal and monthly variable physical quantity which is expressed in terms of number. Both sunspots and solar radio flux are indicators of solar activity for ionospheric ionization level, with higher value during maximum solar activity and low value during minimum solar activity.

Ionosphere is not a stable medium that allows the use of the same frequency throughout the year, or even over 24 hours. It varies with the solar cycle, the seasons and during any given day. These solar activities have their own effects on the total electron content (TEC) (principal indicator of ionospheric variability). As observed from figure 12 (Middle) and (bottom) panels of area graphs in the year of investigation 2015, in general it was found that the sunspot number and solar radio flux are directly proportional to each other.

Now let us investigate how TEC is affected by sunspot number and solar radio flux. figures 13 to 16 , demonstrates daily average sunspot numbers and Sun's radio emission at a wavelength $\lambda=10.7 \mathrm{~cm}$ and frequency $v=2.8 \mathrm{GHz}$. Figure 13 , shows around the beginning of January 11, both sunspot number, radio flux and TEC have higher magnitude and fall down together around January 16, 2015. Again from January $16-25$ both the sunspot number and solar radio flux shows moderate values. After January 26, both sunspot number and solar flux increases to maximum and medium values respectively. A similar phenomenon has occurred before January 11. Here we can see that the value of TEC also increases and decreases in line with the values of these parameters on those corresponding days as depicted from the contour plot.

From figure 14, we have seen that as sunspot number and solar flux are both increased together on March 30 and in the same corresponding days the TEC also increased. We have seen maximum TEC value during March, 2015 and during this time the sunspot number and solar 
radio flux are fair but there are geomagnetic storms during this month. We will see effects of geomagnetic storm days in this month and other similar geomagnetic storm days on other months on the next section. Figure 15 shows from May 10 - 15 both sunspot number, radio flux and TEC have great magnitude together and fall down between May 20 and 30, 2015. Similar with the previous days of months, during August 2015 the variation of TEC in line with the solar activity indices also observed.

In general from figures 13 to 16 , maximum TEC value is formed during spotted days while minimum TEC value is formed during spotless days. For the day exhibits maximum sunspot numbers and solar radio flux, the TEC value is also maximum. It is highly relevant for ionospheric effects. Jee et al. (2005) reported the direct control of the solar activity on the ionization level, with higher values during a high solar activity period and low value during a low solar activity period. We deduce that, during the period of low or high sunspot number, the provided GPS ionospheric TEC builds up slowly or quickly.

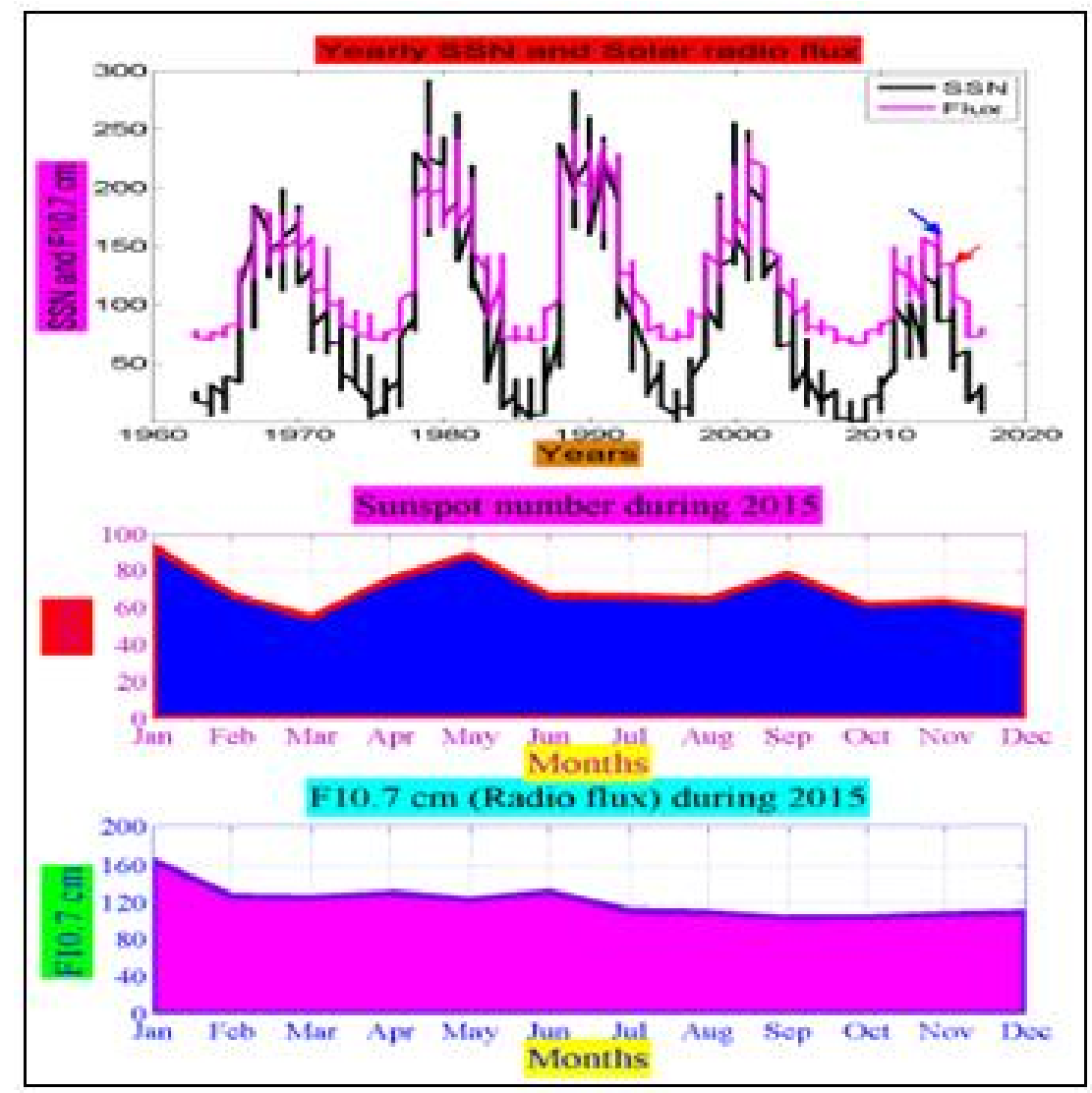

Figure 12. Sunspot number and solar radio flux during (1963 - 2017) (top), sunspot number during 2015 (Middle) and Solar radio flux (bottom) in 2015. 


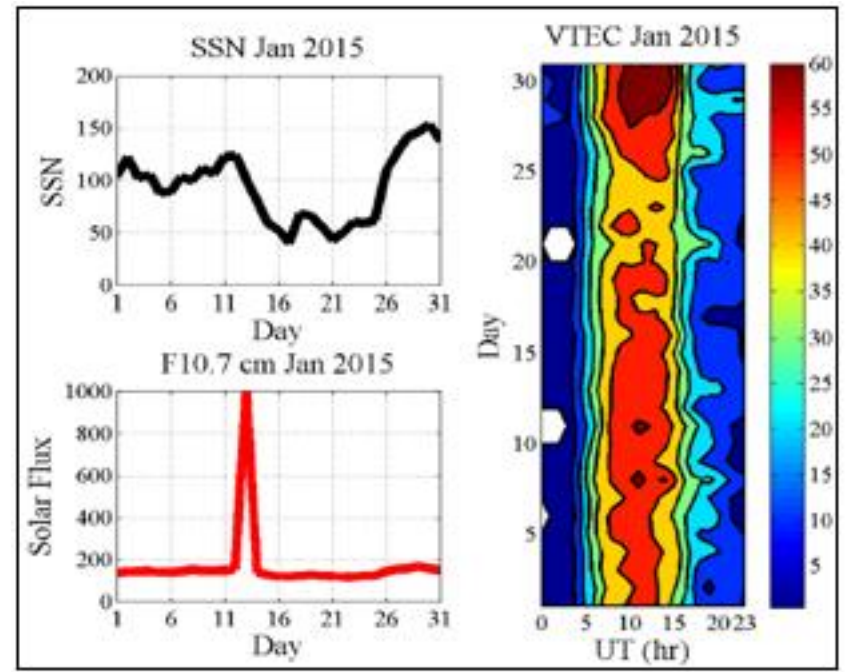

Figure 13. Sunspot number, Solar radio flux and VTEC during January of 2015.

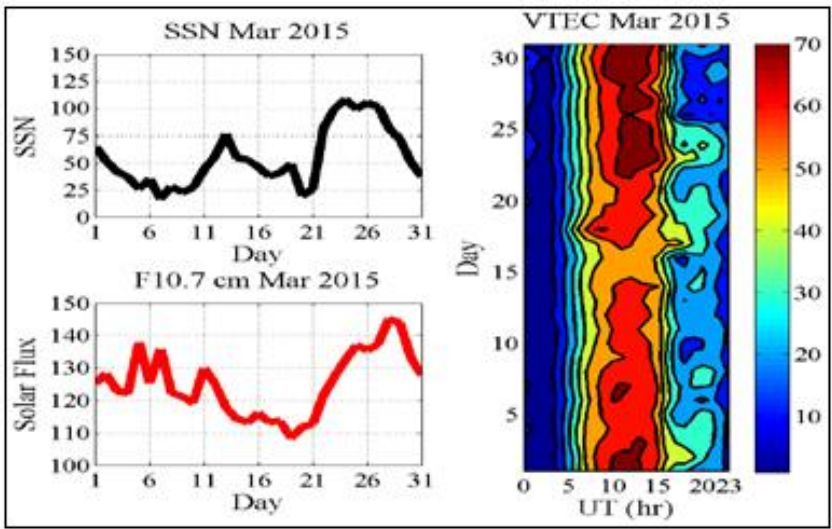

Figure 14. Sunspot number, Solar radio flux and VTEC during March of 2015.

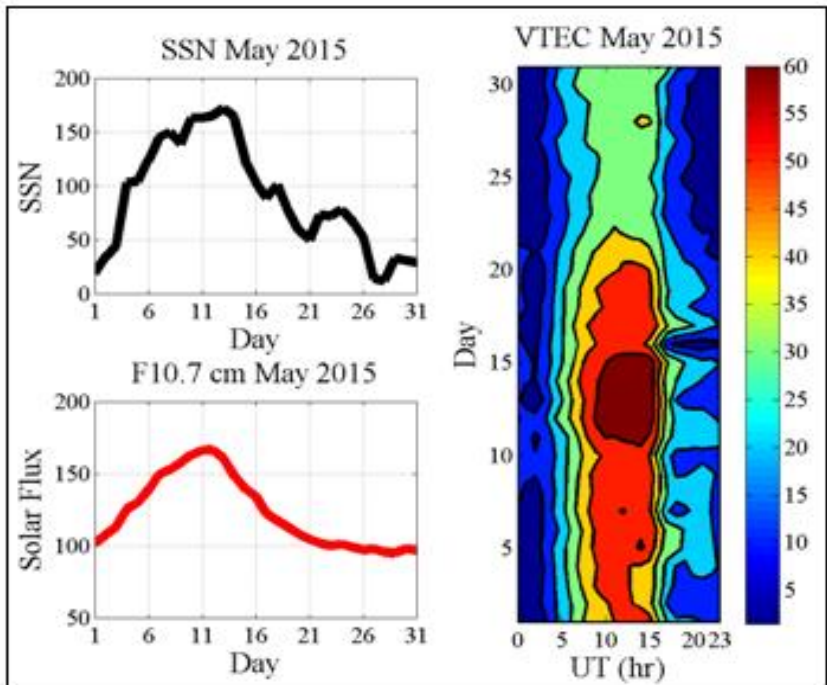

Figure 15. Sunspot number, Solar radio flux and VTEC during May of 2015. 


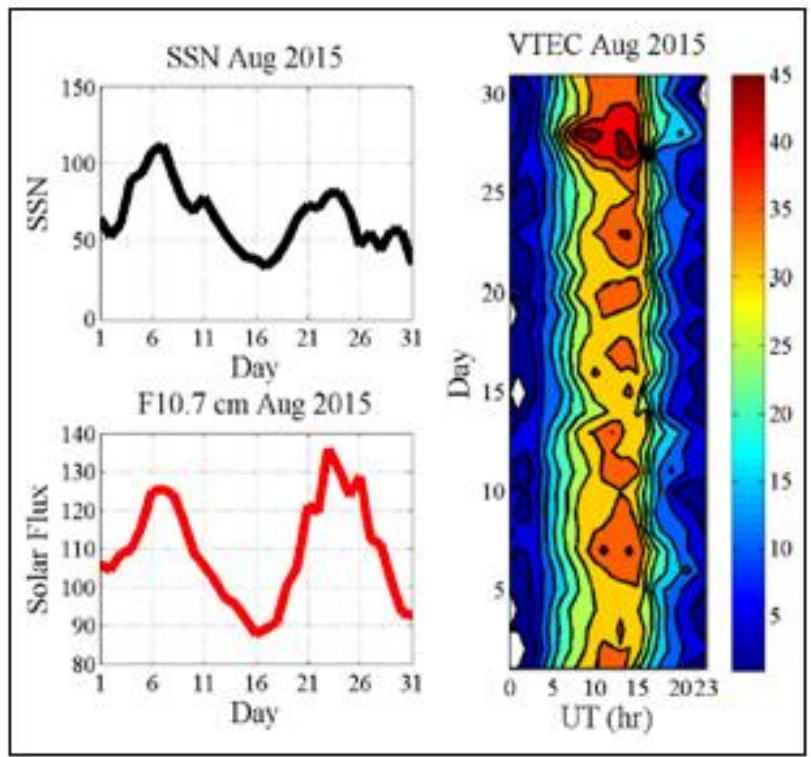

Figure 16. Sunspot number, Solar radio flux and VTEC during August of 2015.

\subsection{Variation of TEC during geomagnetic storms}

The Earth's ionosphere responds dramatically to geomagnetic storms coupling the neutral thermosphere with the overlying magnetosphere. Geomagnetic disturbances are caused either when the interplanetary magnetic field (IMF) is directed south ward or when the magnetosphere of the Earth is stroked by solar wind. On sun spotted day more solar electromagnetic radiation and energetic particles such as CME, solar are, X-ray, EUV are bombarding the ionosphere to have large magnitude of TEC. Strong solar flares could be, associated with cosmic ray and it disrupts radio communication on Earth, the intense X-ray and EUV radiation emitted during the flares heats the atmosphere, disrupting communication and altering Satellites orbits (Schunk and Raitt, 1980)

The size of a geomagnetic storm is classified as moderate when Dst is between $-50 \mathrm{nT}$ and - $100 \mathrm{nT}$, intense storm between - $100 \mathrm{nT}$ and - $250 \mathrm{nT}$ and super storm below - $250 \mathrm{nT}$. Super storms have an annual variation, with higher occurrence around the equinoxes. Most super storms have a short main phase duration $(10-20) \mathrm{hr}$. But there are cases more complex, with main phase lasting more than 40 hours. The Ap is computed every 24 hrs and during quiet periods just above zero but may rise up to $400.0<\mathrm{Ap}<10 \mathrm{nT}$ shows no disturbance and Ap $>10$ $\mathrm{nT}$ shows there is a geomagnetic disturbance. 
From figures 17 to 20 the left panels show VTEC versus time while the right panels show Dst versus time. On day March 17 and 18 with Dst values of equinox time reached - $222 \mathrm{nT}$ and - 189 nT respectively, and on June 23 and August 27, on summer solstice, the Dst values of - 204 nT and - 92 nT respectively. Similarly on November 10 of winter solstice, the Dst values reached - $58 \mathrm{nT}$ at 1400 UT during the main phase of the geomagnetic storm. The Dst values measured on March 17 - 18 and June 23 shows intense geomagnetic disturbance and moderate during August 27 and November 10. The effect of a geomagnetic storm on the ionospheric TEC has been studied by many researchers. The geomagnetic activities have both negative and positive storm effect. The TEC response to the storm depends on the universal time of sudden storm commencement (SSC).

From figure 18, the value of TEC observed at June 23, 2015 was high relative to other undisturbed days in the month. This phenomenon of the TEC variation comes due to SSC in the day local time, in which the high value of interplanetary geomagnetism recorded from this particular day (June 23) and the other months of disturbed days of 2015 as really supported byAp index bar graph. As shown in figure 21, there are different kinds of SSCs of geomagnetic storms. From these different SSC we took some days from international disturbed ( IDD ) and undisturbed ( IQD ) days at which strong geomagnetic storm is formed and no geomagnetic storm was happened respectively depending on Dst indices. Diurnal and disturbance-dependent change in the ionospheric TEC depends on Space Weather. Therefore, there is strong relationship between geomagnetic storm and variation in the ionospheric TEC.

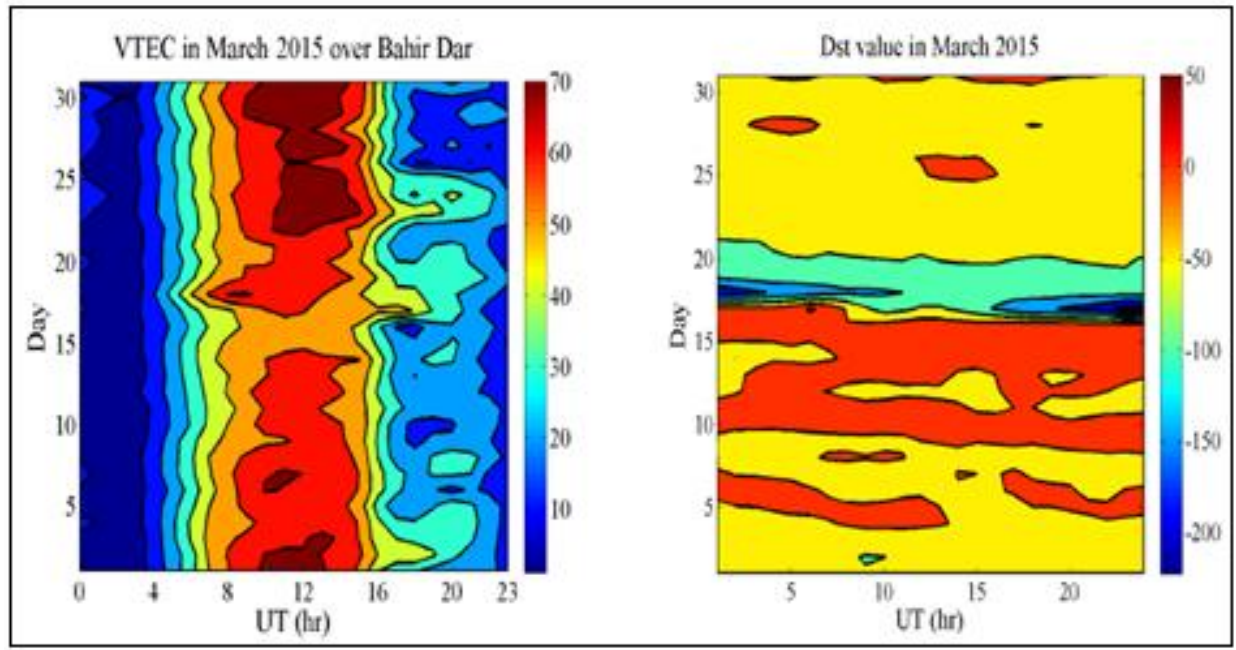

Figure 17. Diurnal variation of TEC and Dst value at Bahir Dar Station in March, 2015. 


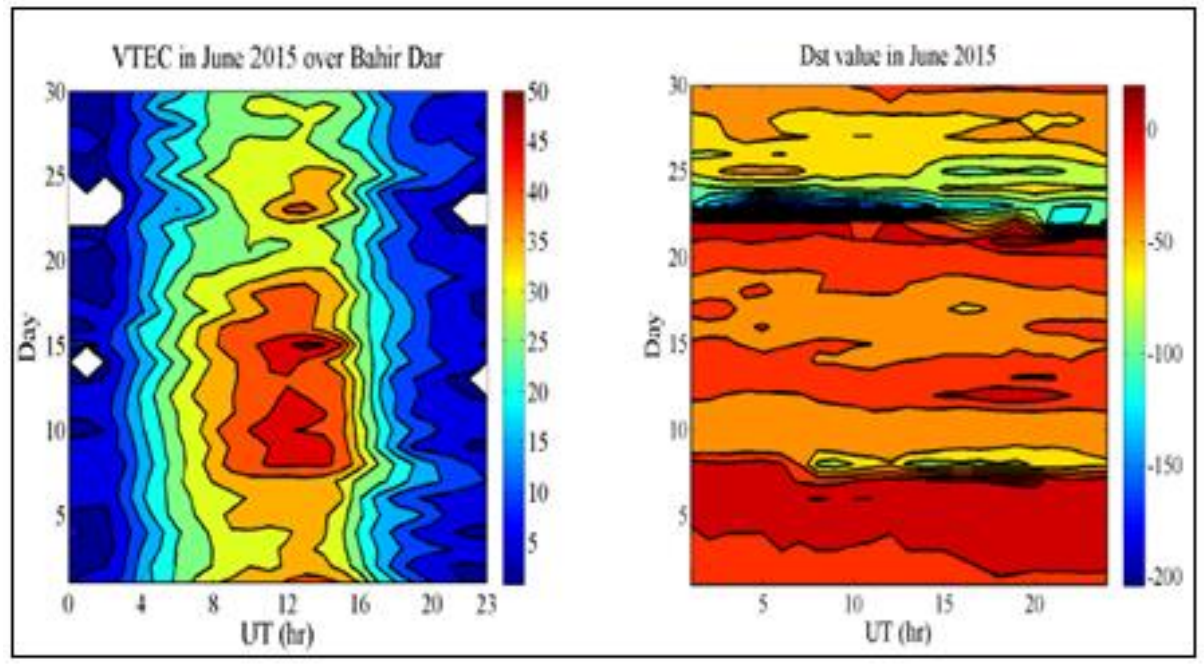

Figure 18. Diurnal variation of TEC and Dst value at Bahir Dar Station in June, 2015.
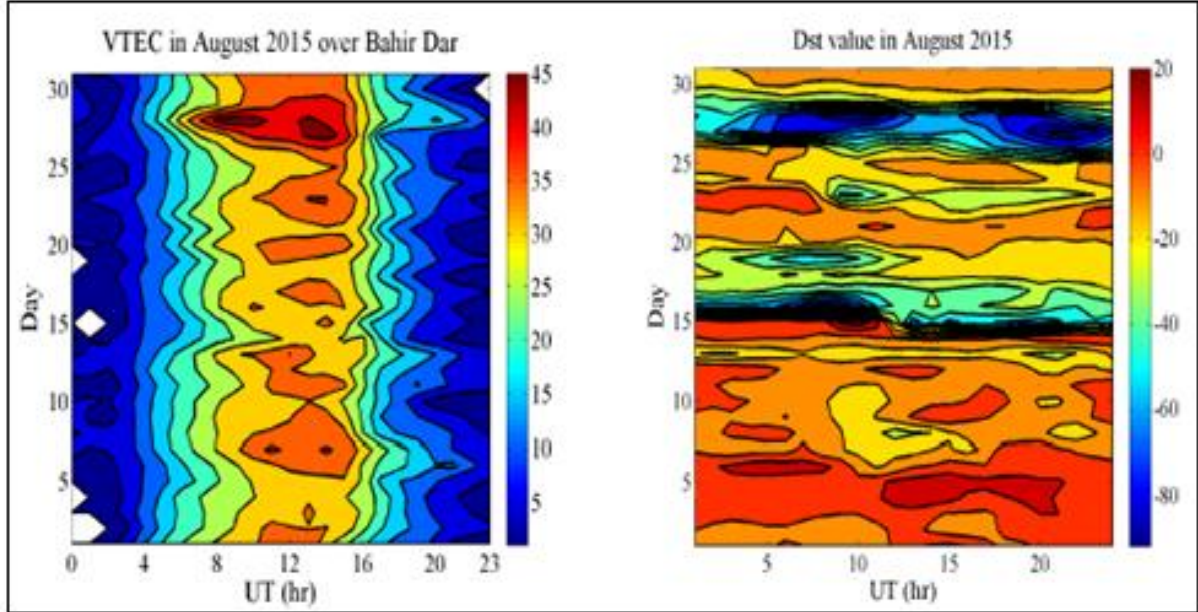

Figure 19. Diurnal variation of TEC and Dst value at Bahir Dar Station in August, 2015.

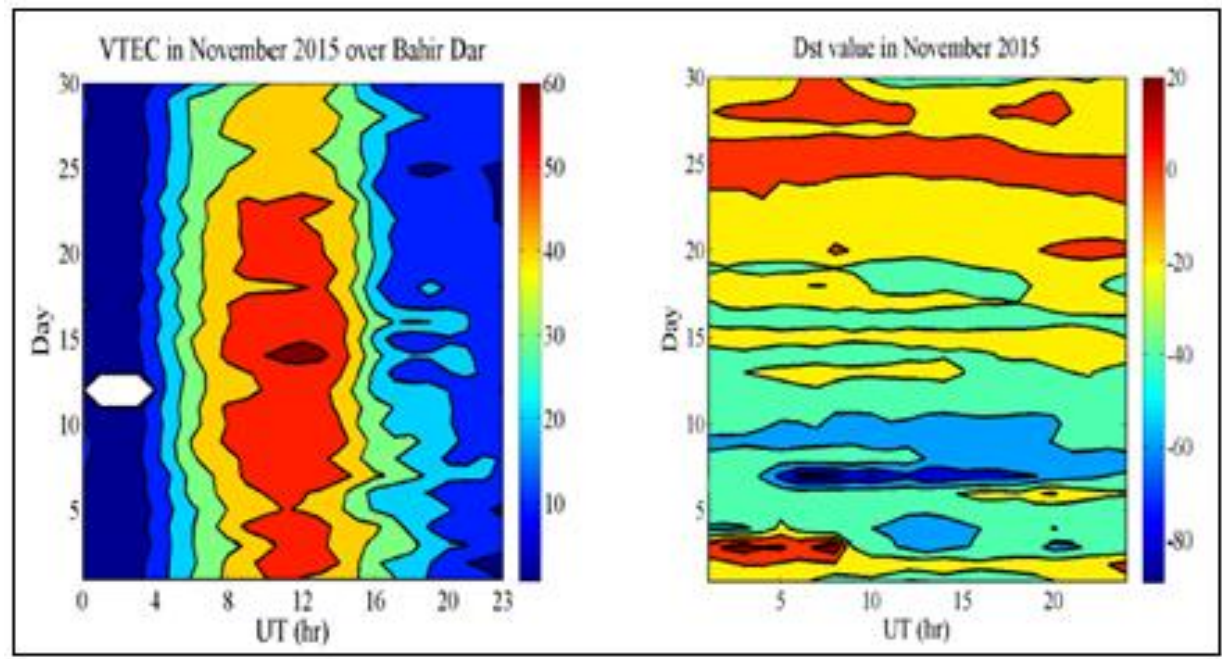

Figure 20. Diurnal variation of TEC and Dst value at Bahir Dar Station in November, 2015. 


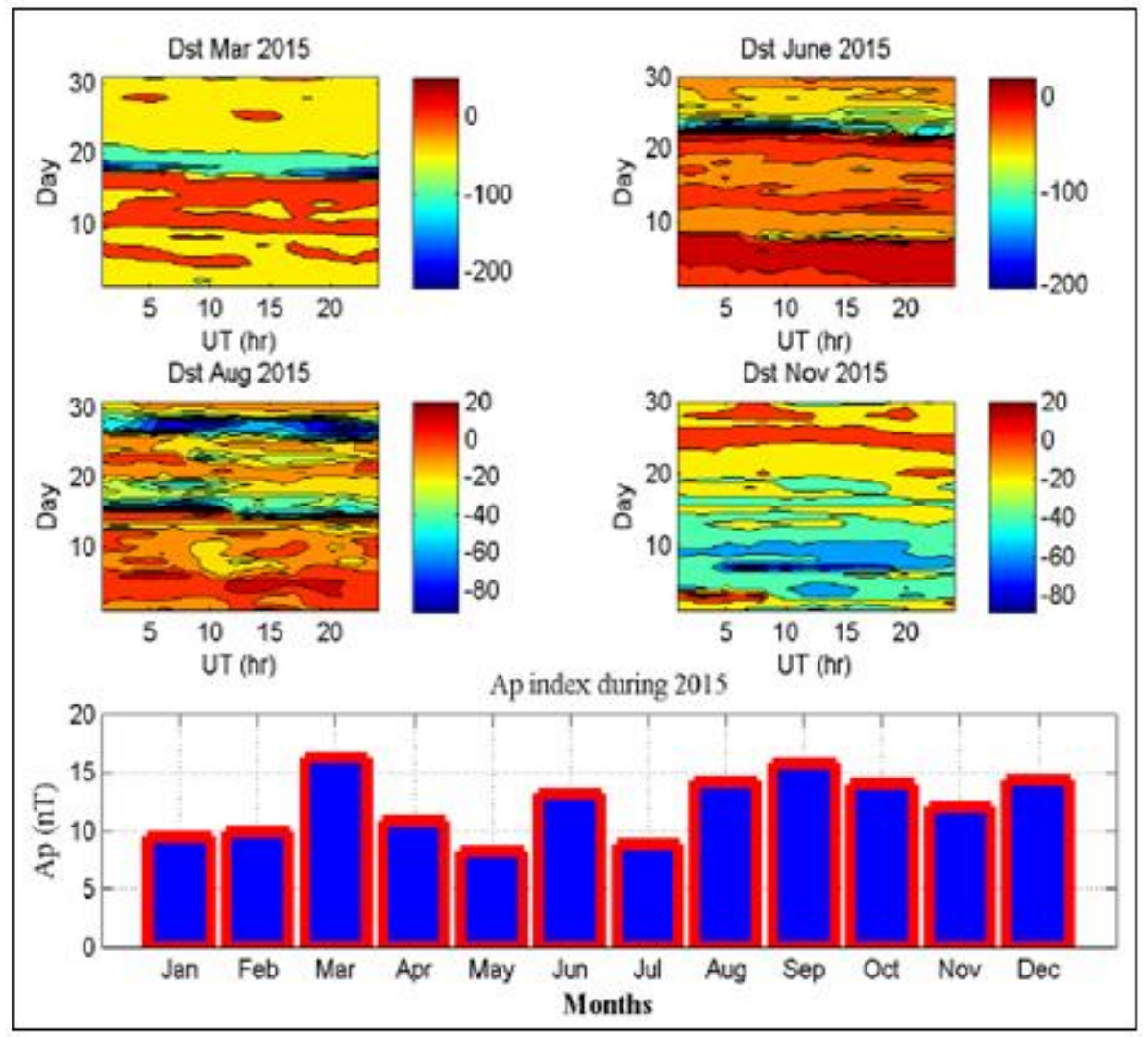

Figure 21. Dst index of March, June, August and November and the corresponding Ap index of 2015.

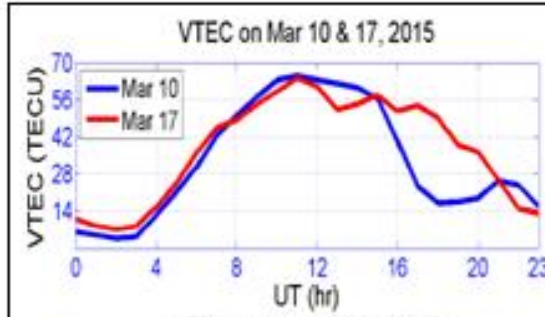

VTEC on June $20 \& 23,2015$

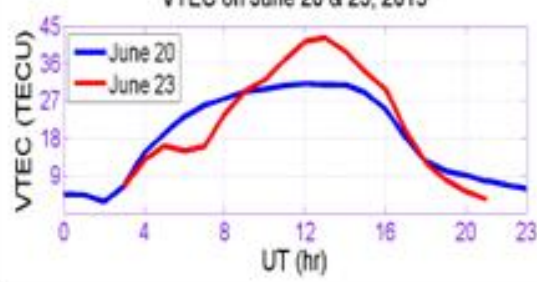

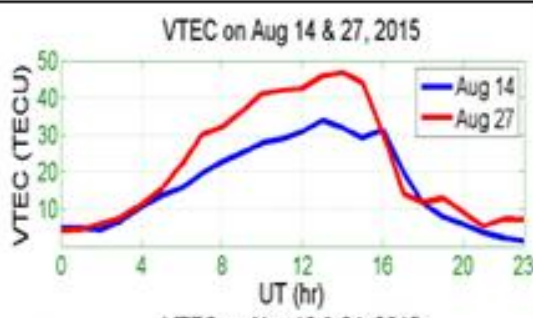

VTEC on Nov $10 \& 24,2015$

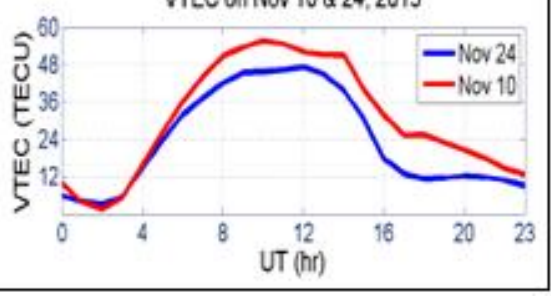

Figure 22. TEC values during disturbed and undisturbed days at Bahir Dar, Ethiopia during 2015.

Figure 22 shows TEC values during IDD's and IQD's at Bahir Dar station during 2015. In these figures, X-axes shows hour universal Time (UT) and Y-axes shows TEC values in TECU. The red lines in figure 22 depict TEC values obtained during disturbed days while the blue lines 
depict TEC values obtained during undisturbed days. During June and August the TEC value on disturbed days is greater than the TEC value on undisturbed days. The TEC values on disturbed days of June 23 (doy=174) and August 27 (doy $=239)$ are 42.21 TECU at 1400 UT and 46.8 TECU at 1500 UT respectively while on undisturbed days of June 20 (doy =171) and August 14 $($ doy $=226)$ are 30.89 TECU at $1400 \mathrm{UT}$ and $33.90 \mathrm{TECU}$ at $1400 \mathrm{UT}$. The maximum values in TEC during November $10(\mathrm{doy}=314)$, is $55.68 \mathrm{TECU}$ at 1100 UT but during November 24 (doy =328), 47.17 TECU at $1300 \mathrm{UT}$.

Similar with June and August the TEC value during November $10($ doy $=314)$ is greater than that of November 24 (doy $=328)$. Here we observe that TEC value changes irregularly for disturbed days as compared to undisturbed days. The maximum values in TEC during March 10 (doy $=69$ ), is 65.14 TECU at 1200 UT but during March 17 (doy =76), 63.96 TECU at 1200 UT. Contrary to the aforementioned days of months result, the value of TEC during March 10 $($ doy $=69)$ is greater than that of March $17($ doy $=76)$.

In Addis Ababa station as shown in figure 23 due to the in availability of the data on the corresponding days of observation like on Bahir Dar station on March, August and November, we selected the next IQD's and IDD's on these days of months by keeping the phenomena on June as already done by that day due to the data availability. Here also the red lines depict disturbed days while blue lines depict undisturbed days. As observed here the value of TEC on August 23 and June 20 is greater than June 20 and August 24 respectively. The value of TEC on March 30 and November 25 is greater than March 18 and November 7, respectively.

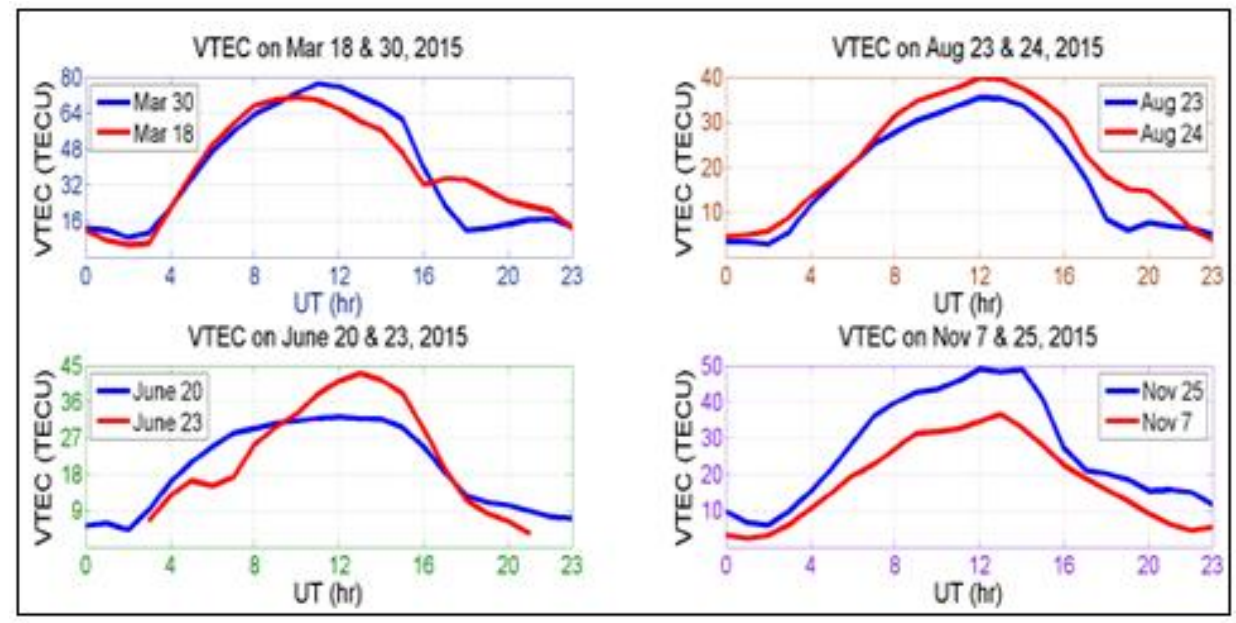

Figure 23. TEC values during disturbed and undisturbed days at Addis Ababa, Ethiopia during 2015. 
Generally, we observe that in both stations the value of TEC is maximum during disturbed days as compared to undisturbed once. In a different case the value of TEC is maximum on undisturbed days of March and November, 2015 as compared to disturbed days as observed from the study areas found in Ethiopia, East Africa. Geomagnetic storms may be positive or negative in regarding to their effects on TEC that is the value of TEC increases due to positive while decreases due to negative geomagnetic storms. So we showed that the storms occurred during March $17-18$ and November 25 are negative storms while the rest days of geomagnetic storms are positive storms since they increase the value of TEC as compared to other undisturbed days of the observed months. When TEC is maximized /minimized by storms in the ionosphere the radio communication will also be disrupted. In all aspects of the observation we saw that the variation of TEC of the ionosphere results due to Space Weather effects.

\section{CONCLUSIONS}

The results show that the median TEC varies from a pre-dawn minimum to an afternoon maximum and then decreases. The daily variation of ionospheric TEC is maximum on the days of equinox and winter while minimum on days of summer seasons in both stations.In all the results obtained the diurnal variations show a maximum occurring of TEC between $0900-1500$ UT, and short-lived minimum in TEC occurred between 1600 - 0700 UT. During February, March and April 2015 the magnitude of the TEC is better than the rest days of other months at 0000 - 2300 UT. The three months (March, April and February) TEC value during 2015 at both stations is extremely differ from other months TEC value. In the whole seasons the maximum value of TEC leads first in Addis Ababa.

The value of TEC shows increment and decrement in line with the values of solar activity parameters (sunspot number and solar radio flux). We deduce that, during the period of low or high sunspot number and solar radio flux, the provided GPS ionospheric TEC builds up slowly or quickly. Geomagnetic storm has occurred on June 23, 2015. The Dst values for the day shows that it is an intense intensity geomagnetic storm. We observed the value of TEC is greater during disturbed days than undisturbed once. In a different case the value of TEC is maximum on undisturbed day of March and November, 2015 as compared to disturbed days. We showed that the storms occurred during March 17 - 18 and November 25 are negative storms while the rest 
days of geomagnetic storms are positive storms. When TEC is maximized /minimized by storms in the ionosphere the radio communication will also be disrupted. In all aspects of the observation we saw that the variation of TEC of the ionosphere results due to Space Weather effects.

\section{ACKNOWLEDGEMENTS}

The authors acknowledge all data providers NASA, World Data Center (WDC), Kyoto for Dst index, WDC and UNAVCO. The authors of cited journals and books are also acknowledged.

\section{CONFLICT OF INTERESTS}

There are no conflicts of interest.

\section{REFERENCE}

Abdu, A \& Brum, C. G. M. 2009. Electrodynamics of the vertical coupling processes in the atmosphere-ionosphere system of the low latitude region. Earth Planets Space, 61: 385395.

Abdullah, M., Strangeways, H.J \& Walsh, D.M.A. 2009. Improving ambiguity resolution rate with an accurate ionospheric differential correction. Journal of Navigation, 62(1): 151166.

Adolph S. J. 1985. Handbook of Geophysics and the Space Environment. United States.

Arnold, H. 2007. The Sun and Space Weather. Volume 347, Springer, $2^{\text {nd }}$ edition, ISBN-10 14020-5603-6.

Bagiya, S., Iyer, K. N.,Joshi, H. P., Thampi, S. V., Tsugawa, T., Ravindran, S., Sridharan, R \& Pathan, B. M. 2011. Low-latitude ionospheric-thermospheric response to storm time Electro-dynamical coupling between high and low latitudes. Journal of Geophysical Research, 116(A1): A01303.

Coster, A \& Komjathy, A. 2008. Space weather and the global positioning system. doi:10.1029/2008SW000400.

Denton, M. H., Borovsky, J. E., Skoug, R. M., Thomsen, M. F., Lavraud, B., Henderson, M. G., Mc Pherron, R. L., Zhang, J. C \& Liemohn, M. W. 2006. Geomagnetic storms driven by ICME- and CIR-dominated solar wind. Geophysical Research, 111: A07S07, doi:10.1029/2005JA011436. 
Hintsa, G \& Abraha, G. 2017. Global Variations of Ionospheric Total Electron Content (TEC) Derived from GPS Global Ionospheric Maps. Momona Ethiopian Journal of Science, 9(2): 141-161.

Jakowski, N., Heise, S., Wehrenpfenning, S., Schluter, S \& Reimer, R. 2002. Gps/glonass-based tec measurements as a contribution for space weather forecast. Atmos. and Sol. Terr. Phys, 64: 729-735.

Jee, G., Schunk, R. W \& Scherliess, I. 2005. Comparison of IRI-2001 with topex TEC measurements. Sol. Terr. phys., 67: 365-380.

Klobuchar, J.A., 1987. Ionospheric time-delay algorithm for single-frequency GPS users. IEEE Trans. Aerosp. Electron. Syst., AES-23, 325-331.

Kelly, M. C. 1989. The Earth's Ionosphere, plasma physics and electrodynamics. Academic press, INC, New York.

Klobuchar, J.A., 1987. Ionospheric time-delay algorithm for single-frequency GPS users. IEEE Trans. Aerosp. Electron. Syst., AES-23, 325-331.

Maruyama, N., Watanabe, S \& Fuller-Rowell, T. J. 2003. Dynamic and energetic coupling in the equatorial ionopsphere and theremosphere. J. Geophys. Res., 108: A11, 1396, doi:10.1029/2002JA009599.

Michael, C. K. 2009. The earth's ionosphere plasma physics and electrodynamics. International Geophysics Series, Academic Press, Inc.

Rabiu, A. B., Mamukuyomi, A. I \& Joshua, E. O. 2007. Variability of equatorial ionosphere inferred from geomagnetic field measurements. Bull. Astr. Soc. India, 35: 607-618.

Rama Rao, P. V. S., Gopi Krishna, S., Niranjan, K \& Prasad, D. S. V. V. D. 2006. Temporal and spatial variations in TEC using simultaneous measurements from the Indian GPS network of receivers during the low solar activity period of 2004-2005. AnnalesGeophys., 24(12): 3279-3292.

Rastogi, R. G., Chandra, H \& James, M. E. 2008. Characteristics of equatorial electrojet current in the central regions of South America. Earth Planets Space, 60: 623-632.

Ke, Su., Shuanggen, Jin \& Hoque, M. M. 2019. Evaluation of Ionospheric Delay Effects on Multi GNSS Positioning Performance. Remote Sensing. doi:10.3390/rs11020171.

Schunk, R. W \& Raitt, W. J. 1980. Atomic nitrogen and oxygen ions in the daytime high latitude F region. J. Geophys. Res., 85: 1255. 
Tyagi, T. R. 1974. Electron content and its variation over lindau. Atmos. Sol-Terr. Phys (UK), 36: $475-487$.

Tsurutani, B. T. 2000. Solar/interplanetary plasma phenomena causing geomagnetic activity at earth. IOS Press, 273p.

Yizengaw, E., Moldwin, M. B., Dyson, P. L \& Essex, E. A. 2007. Using Tomography of GPSTEC to Routinely Determine Ionospheric Average Electron Density Profiles, $J$. Atmos.Solar-Terr. Phys., 69: 314-321.

Yu, You., WAN, Wei-Xing., Li-Bo, L \& Bi-Qiang, Z. 2009. A global ionospheric TEC perturbation index, China. 